\title{
Healthy Super Mario: Tap Your Stomach and Shout for Active Healthcare
}

\author{
Jaewook Jung, Shin Kang, Hanbit Park, and Minsoo Hahn \\ Digital Media Laboratory, KAIST, \\ 517-10 Dogok-dong, Gangnam-gu, Seoul 135-854, S. Korea \\ \{jaewookjung, kangshin12, han_bit,mshahn2\} @kaist.ac.kr
}

\begin{abstract}
The purpose of game provides fun and enjoyment to users. Most of the game, however, has physical dysfunction with providing fun experience. As a solution about this problem, the body movement based game like Nintendo Wii was presented. However, it just prevents the physical dysfunction by adopting body movement as game input and requires the special controller to play a game. In this research, we suggest a new game input style which is tapping stomach and shouting. It is not only preventing the dysfunction but also promoting health.
\end{abstract}

Keywords: Game interface, Interaction style, Active healthcare.

\section{Introduction}

A physical dysfunction has been occurred with the growth of game industry [1]. As a physical dysfunction by game, there are Visual Display Terminal (VDT) syndrome, Spinal curvature, and Carpal Tunnel syndrome. To solve the problems, the exertion game interface like Nintendo Wii or Dance Dance Revolution [DDR] was developed [2], but it just prevented the physical dysfunction. We suggest two new game input methods for active healthcare beyond just preventing ill health. One is tapping stomach with both hands. Some medical research was shown that tapping stomach by both hands promotes the secernent of digestive fluid and the circulation of the blood. In other words, it promotes a digestive process [3]. The other style is shouting like a kendo player. Shouting like a kendo or taekwondo player is effective in reducing stress and improving concentration [4]. We suggest a new game interface that detects tapping the stomach by applying digital image processing (DIP) technology and measures the shouting level by digital signal processing (DSP) technology. We also present new athletic game, Health Super Mario, with the suggested interface.

\section{Game Interface and Healthy Super Mario}

The suggested interface consists of one webcam and one normal PC. The webcam detects the tapping stomach by both hands and counts the number of times tapping the stomach occurs in interval of $500 \mathrm{~ms}$. The green colored ellipses in Figure 1-(a) is 
the tapping area and intestines are located in Figure 1-(b). The microphone of the webcam detects shouting by a player and measures the volume of shouting.

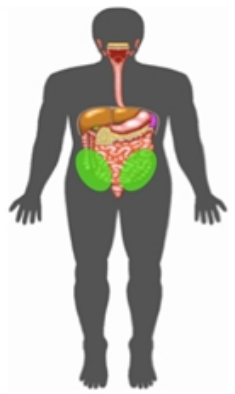

(a)

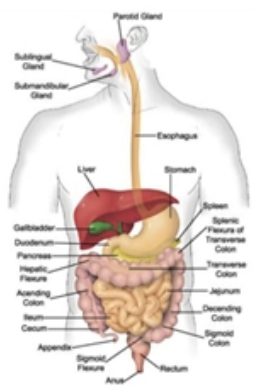

(b)

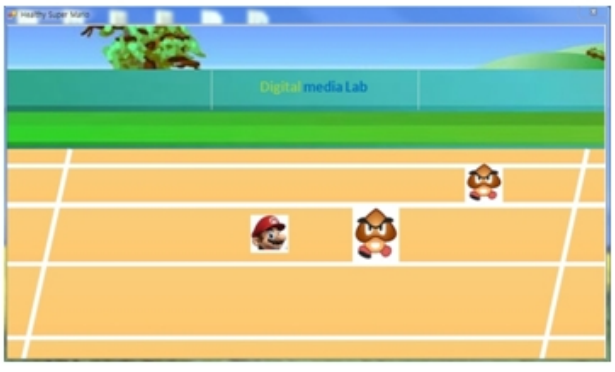

(c)

Fig. 1. (a) An anatomical chart, (b) A digestive system, and (c) The screenshot of Healthy Super Mario

Figure 1-(c) shows that Health Super Mario athletic game played by the suggested interface. Mario runs and jumps to avoid Goomba, an enemy character. The running speed is proportional to the number of tapping stomach per unit time and Mario jumps by shouting.

\section{Conclusion}

Many office workers or youth immediately play an online game or web surfing after eating a meal. This behavior has a bad influence on a digestive process. In this study, we suggest new input style that uses tapping stomach and shouting as game input. It aims to prevent the physical dysfunction and builds up health by changing a game style in life. As a result, the suggested input style is an entertainment system for active health.

\section{References}

1. Jung, J., Hahn, H., Yamazaki, T.: Game Interface Design Guidelines to Solve the Dysfunction of an Online Game. In: 21st International Symposium Human Factors in Telecommunication, pp. 359-366. Prentice Hall, Englewood Cliffs (2008)

2. Wii, N.: http: / / www . nintendo.com/wii

3. Kim, J., Park, J.: The Effect of Abdominal Meridian Massage on the Degree of Constipation among the Patients with Immobility after Orthopedic Surgery. Clinical Nursing Research 12(2), 63-72 (2006)

4. Kang, K., Yun, J.: The Effects of shouting on Motor Neural Adaptation and Maximum Isometric Contraction. Korean Journal of Sport Science 12(4), 85-93 (2001) 